\title{
LINEARLY DEPENDENT SUBSETS OF SEGRE VARIETIES
}

\author{
E. BALLICO
}

\begin{abstract}
We study the linear algebra of finite subsets $S$ of a Segre variety $X$. In particular we classify the pairs $(S, X)$ with $S$ linear dependent and $\#(S) \leq 5$. We consider an additional condition for linear dependent sets, i.e. that no two of their points are contained in a line of $X$, and get far better lower bounds for $\#(S)$ in term of the dimension and number of the factors of $X$. In this discussion and in the classification of the case $\#(S)=5, X \cong \mathbb{P}^{1} \times \mathbb{P}^{1} \times \mathbb{P}^{1}$ we use the rational normal curves contained in $X$.
\end{abstract}

\section{INTRODUCTION}

Let $K$ be a field, and let $k$ and $n_{i}$ be positive integers, with $1 \leq i \leq k$. Set $Y:=\prod_{i=1}^{k} \mathbb{P}^{n_{i}}$, the multiprojective space with $k$ non-trivial factors of dimension $n_{1}, \ldots, n_{k}$. Set $r:=-1+\prod_{i=1}^{k}\left(n_{i}+1\right)$. Let $\nu: Y \rightarrow \mathbb{P}^{r}$ denote the Segre embedding of the multiprojective space $Y$. Thus $X:=\nu(Y)$ is a Segre variety of dimension $n_{1}+\cdots+n_{k}$. It was introduced by Corrado Segre in 1891 ([14]). See $[10$, Ch. 25] for its geometry over a finite field. In the last 30 years this variety has had a prominent role in the applied sciences, because it is strongly related to tensors and it was realized that tensors may be used in Engineering and other sciences ([12]).

Let $S \subset Y$ be a finite subset. Set

$$
e(S):=h^{1}\left(\mathcal{I}_{S}(1, \ldots, 1)\right)=\#(A)-1-\operatorname{dim}\langle\nu(A)\rangle,
$$

where \langle\rangle denotes the linear span. The minimal multiprojective subspace $Y^{\prime}$ of $Y$ containing $S$ is the multiprojective space $\prod_{i=1}^{k}\left\langle\pi_{i}(S)\right\rangle \subseteq Y$, where $\left\langle\pi_{i}(S)\right\rangle$ denote the linear span of the finite set $\pi_{i}(S)$ in the projective space $\mathbb{P}^{n_{i}}$. We say that $Y^{\prime}$ is the multiprojective subspace generated by $S$ and that $S$ is non-degenerate if $Y^{\prime}=Y$. We say that $S$ is linearly independent if $\nu(S) \subset \mathbb{P}^{r}$ is linearly independent. By the definitions of Segre embedding and of the integer $e(S)$ we have $\operatorname{dim}\langle\nu(S)\rangle=\#(S)-1-e(S)$. In particular, $S$ is linearly dependent if and only if $e(S)>0$. We say that $S$ is a circuit if $S$ is linearly dependent, but every proper subset of $S$ is linearly independent.

Everything said up to now uses only the linear structure of the ambient $\mathbb{P}^{r}$. Now we describe the new feature coming from the structure of $Y$ as a multiprojective space, in particular the structure of linear subspaces contained in the Segre variety $\nu(Y)$. We say that a finite set $S \subset Y$ is minimal if there is no line $L \subset \nu(Y)$ such that $\#(\nu(S) \cap L) \geq 2$. If $\#(\nu(S) \cap L) \geq 3$, then $S$ is not linearly independent. However, a non-minimal finite set $S$ may be linearly independent; for example, take as $S$ two points such that the line $\langle\nu(S)\rangle$ is contained in $\nu(Y)$. When $S$ is linearly independent there is no $A \subset Y$ such that $\#(A)<\#(S)$ and $\langle\nu(A)\rangle \supseteq\langle\nu(S)\rangle$. If $S$ is not minimal, say there is $L \subset Y$ such that $\nu(L)$ is a line and $L \cap S \supseteq\{a, b\}$ with $a \neq b$, there is $o \in L$ such that

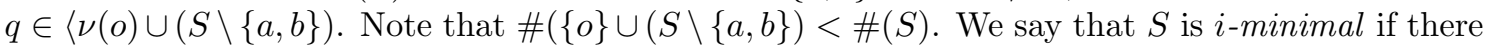
is no curve $J \subset Y$ such that $\nu(J)$ is a line, $\#(J \cap S) \geq 2$ and $J$ is mapped isomorphically into the $i$-th factors of $Y$, while it is contracted to a point by the projections onto the other factors of $Y$. The finite set $S$ is minimal if and only if it is $i$-minimal for all $i$. The minimality condition is in general different from the assumptions needed to apply Kruskal's criterion to two subsets $A, B \subset S$ with $A \cup B=S$ and $\#(A)=\#(B)$; see $[2,3,4,5,6,7,11]$.

We classify circuits with cardinality 4 (Proposition 5.2) and give the following classification of circuits formed by 5 points.

Theorem 1.1. Let $\Sigma$ denote the set of all non-degenerate circuits $S \subset Y$ such that $\#(S)=5$. Then one of the following cases occurs:

2010 Mathematics Subject Classification. 14N05; 12E99; 12 F99.

Key words and phrases. Segre varieties.

The author was partially supported by MIUR and GNSAGA of INdAM (Italy). 
(1) $k=1, n_{1}=3$;

(2) $k=2, n_{1}=n_{2}=1$;

(3) $k=2$ and $n_{1}+n_{2}=3$; all $S \in \Sigma$ are described in Example 5.\%;

(4) $k=3, n_{1}=n_{2}=n_{3}=1$; all $S \in \Sigma$ are described in Lemma 5.8; in this case, $\Sigma$ is an irreducible variety of dimension 11 .

All $S \in \Sigma$ in the first two cases listed in Theorem 1.1 are described in Remark 5.5. In the classification of case (4), we use the rational normal curves contained in a Segre variety.

The non-degenerate sets $S$ with $\#(S)=5$ and $e(S) \geq 2$ are also classified; see Proposition 5.6.

The study of linearly dependent subsets of Segre varieties with low cardinality was started in [13].

The author has no conflict of interest.

\section{Preliminaries}

Take $Y=\mathbb{P}^{n_{1}} \times \cdots \times \mathbb{P}^{n_{k}}, k \geq 1, n_{i}>0,1 \leq i \leq k$. Set $r:=-1+\prod_{i=1}^{k}\left(n_{i}+1\right)$. Let $\nu: Y \rightarrow \mathbb{P}^{r}$ denote the Segre embedding of $Y$. We will use the same name, $\nu$, for the Segre embedding of any multiprojective subspace $Y^{\prime} \subseteq Y$. Set $X:=\nu(Y) \subset \mathbb{P}^{r}$. For any $q \in \mathbb{P}^{r}$ the $X$-rank of $q$ is the minimal cardinality of a finite subset $S \subset X$ such that $q \in\langle S\rangle$, where \langle\rangle denotes the linear span. For any $q \in \mathbb{P}^{r}$ let $\mathcal{S}(Y, q)$ denote the set of all $A \subset Y$ such that $\#(A)=r_{X}(q)$ and $q \in\langle\nu(A)\rangle$. In the introduction we observed that $S \notin \mathcal{S}(Y, q)$ for any $q \in \mathbb{P}^{r}$ if $S$ is not minimal.

For any $i \in\{1, \ldots, k\}$, set $Y_{i}:=\prod_{h \neq i} \mathbb{P}^{n_{h}}$ with the convention that $Y_{1}$ is a single point when $k=1$.

Let $\eta_{i}: Y \rightarrow Y_{i}$ denote the projection; it is the map obtained by deleting the $i$-th coordinate of the points of $Y$. Here $h^{0}\left(\mathcal{O}_{Y}(1, \ldots, 1)\right)=r+1$. For any $i \in\{1, \ldots, k\}$, let both $\mathcal{O}_{Y}\left(\varepsilon_{i}\right)$ and $\mathcal{O}_{Y}\left(\hat{\varepsilon}_{i}\right)$ be the line bundle $\mathcal{O}_{Y}\left(a_{1}, \ldots, a_{k}\right)$ on $Y$ with multidegree $\left(a_{1}, \ldots, a_{k}\right)$ : the former has $a_{i}=1$ and $a_{j}=0$ for all $j \neq i$; the latter has $a_{i}=0$ and $a_{j}=1$ for all $j \neq i$. Then $h^{0}\left(\mathcal{O}_{Y}\left(\varepsilon_{i}\right)\right)=n_{i}+1$ and $h^{0}\left(\mathcal{O}_{Y}\left(\hat{\varepsilon}_{i}\right)\right)=(r+1) /\left(n_{i}+1\right)$.

Definition 2.1. Take $S \subset Y$ such that $e(S)>0$. $S$ is said to be strongly essential if $e\left(S^{\prime}\right)=0$ for all $S^{\prime} \subset S$ such that $\#\left(S^{\prime}\right)=\#(S)-e(S)$.

Take an essential set $S \subset Y$ and any $S^{\prime} \subset S$. We have $e\left(S^{\prime}\right)=\max \left\{0, e(S)-\#(S)+\#\left(S^{\prime}\right)\right\}$.

A set $S \subset Y$ with $e(S)=1$ is strongly essential if and only if it is a circuit.

We recall the following lemma ([1, Lemma 2.4]), whose proof works over any algebraically closed field, although it was only claimed over $\mathbb{C}$, or at least over an algebraically closed base field with characteristic 0

Lemma 2.2. Take $q \in \mathbb{P}^{r}$ and finite sets $A, B \subset Y$ irredundantly spanning $q$. Fix an effective divisor $D \subset Y$. Assume that $A \neq B$ and that $h^{1}\left(\mathcal{I}_{A \cup B \backslash D \cap(A \cup B)}(1, \ldots, 1)(-D)\right)=0$. Then $A \backslash A \cap D=B \backslash B \cap D$.

Proof. In [1] there is the default assumption that the base field is $\mathbb{C}$ or at least an algebraically closed field of characteristic 0 . The proof of [1, Lemma 2.5], whose statement implies [1, Lemma 2.4], never uses any assumption on the characteristic of the base field. Now we explain why the statement of Lemma 2.2 over an algebraic closure $\bar{K}$ of $K$ implies the statement over $K$. By assumption, all points of $A$ and $B$ are defined over $K$. The dimension of a linear span of a subset of $\nu(A \cup B)$ is the same over $K$ or over $\bar{K}$. Since the statement of the lemma also uses cohomology groups of coherent sheaves we also need to use that the dimension of the cohomology groups of coherent sheaves on projective varieties defined over $K$ is preserved when we extend the base field $K \subseteq \bar{K}$, because $\bar{K}$ is flat over $K$ ([9, Proposition III.9.3]).

Remark 2.3. Fix an integer $e>0$ and an integral and non-degenerate variety $W \subset \mathbb{P}^{r}$ defined over $\bar{K}$. Since $W(\bar{K})$ is Zariski dense, $r+1+e$ is the maximal cardinality over a finite set $S \subset W(\bar{K})$ such that $\operatorname{dim}\langle S\rangle=\#(S)-1-e$. The same is true if $W$ is defined over $K$ and we require that $S \subset W(K)$ and that $W(K)$ is Zariski dense in $W(\bar{K})$. The minimal such cardinality of any such set $S \subset W(\bar{K})$ is $e+2$ if and only if $W$ contains a line; otherwise it is larger. If $K$ is finite to get the same it is sufficient to assume $\#(K) \geq e+1$ and that the line $L \subset W$ is defined over $K$. The Segre variety has enough lines defined over the base field $K$. 


\section{Linear ALGEBra inside the SEgre VARIETIES}

Take $Y:=\mathbb{P}^{n_{1}} \times \cdots \times \mathbb{P}^{n_{k}}, n_{i}>0$ for all $i$.

Remark 3.1. Fix a finite non-degenerate set $S \subset Y$. Let $h^{1}\left(\mathcal{I}_{S}(1, \ldots, 1)\right)>0$; that is, $\nu(S)$ is linearly independent. Equivalently, $S$ is linearly dependent and $h^{1}\left(\mathcal{I}_{S^{\prime}}(1, \ldots, 1)\right)=0$ for all $S^{\prime} \subsetneq S$; that is, each proper subset of $S^{\prime}$ is linearly independent. Equivalently, let $S \subset Y$ be a non-degenerate circuit. In particular, $h^{1}\left(\mathcal{I}_{S}(1, \ldots, 1)\right)=1$, that is, $\operatorname{dim}\langle\nu(S)\rangle=\#(S)-2$. Since $\mathcal{O}_{Y}(1, \ldots, 1)$ is very ample, so $\#(S) \geq 3$.

Remark 3.2. Take $Y=\mathbb{P}^{n_{1}} \times \cdots \times \mathbb{P}^{n_{k}}$ and set $m:=\max \left\{n_{1}, \ldots, n_{k}\right\}$. Then $m+1$ is the minimum cardinality of a subset of $Y$ generating $Y$, that is, not contained in a proper multiprojective subspace of $Y$. To verify this, take $S$ such that $\operatorname{dim}\left\langle\pi_{i}(S)\right\rangle=\min \left\{n_{i}, \#(S)-1\right\}$ for all $i$.

Example 3.3. Let $S \subset Y$ be a finite linearly independent subset, $S \neq \emptyset$, and set $s:=\#(S)$. Fix $i \in\{1, \ldots, k\}$. We construct another subset $S_{i} \subset Y$ such that $\#\left(S_{i}\right)=s+1$ and $\#\left(S_{i} \cap S\right)=s-1$ in the following way and discuss when (assuming $s \leq r) S_{i}$ is linearly independent. Fix $o \in S$ and $i \in\{1, \ldots, k\}$. Take a line $L \subseteq \mathbb{P}^{n_{i}}$ containing $o_{i}$. Fix two points $o_{i}^{\prime}, o_{i}^{\prime \prime} \in L \backslash L \cap \pi_{i}(S)$. Let $o^{\prime}$ be the only point of $Y$ with $\pi_{i}\left(o^{\prime}\right)=o_{i}^{\prime}$ and $\left.o^{\prime \prime}\right)$ the only point with. $\pi_{i}\left(o^{\prime \prime}\right)=o_{i}^{\prime \prime}$ and $\pi_{j}\left(o^{\prime}\right)=\pi_{j}\left(o^{\prime \prime}\right)=\pi_{j}(o)$ for all $j \neq i$. Set $S_{i}:=(S \backslash\{o\}) \cup\left\{o^{\prime}, o^{\prime \prime}\right\}$. Then $\#\left(S_{i}\right)=\#(S)+1$. By assumption, $\operatorname{dim}\langle\nu(S)\rangle=s-1$. Let $D \subseteq D^{\prime} \subseteq Y$ be the multiprojective subspace of $Y$ with $\pi_{j}(o)$ as their projection for all $j \neq i, \pi_{i}(D)=L$ and $\pi_{i}\left(D^{\prime}\right)=\mathbb{P}^{n_{i}}$. The set $S_{i}$ is linearly independent for general $o_{i}^{\prime}, o_{i}^{\prime \prime} \in L$ and for general $o_{i}^{\prime}, o_{i}^{\prime \prime} \in L$ and for a general line $L \subseteq \mathbb{P}^{n_{i}}$ if and only if $\operatorname{dim}\langle\nu(S \cup D)\rangle \geq s$. Now assume $s \neq r+1$ and set $E:=S \backslash\{o\}$. Take a general $\left(a_{1}, \ldots, a_{k}\right) \in Y$, and a general line $J \subset \mathbb{P}^{n_{i}}$ such that $a_{i} \in J$. Let $T \subset Y$ be the irreducible curve with $\pi_{h}(T)=a_{h}$ for all $h \neq i, \pi_{i}(T)=J$ and $\pi_{i \mid T}: T \rightarrow J$ an isomorphism. Then $L:=\nu(T)$ is a line and $\operatorname{dim}\langle L \cup \nu(E)\rangle=s$. We describe in Lemma 3.5 and Remark 3.6 some cases in which we may take $\nu(o) \in L$. For any linearly independent set $A \subset Y$ with $\#(A) \leq r$, there is $o \in Y \backslash A$ such that $A \cup\{o\}$ is linearly independent, because $\nu(Y \backslash A)$ spans $\mathbb{P}^{r}$.

The set $S_{i}$ constructed in Example 3.3 is an elementary increasing of $S$ in the $i$-th factor or an $i$-elementary increasing of $S$. We say that the set $S$ is obtained from $S_{i}$ by an elementary decreasing of the non-minimal set $S_{i}$ in the $i$-th direction or by an $i$-elementary decreasing.

Note that $S_{i} \subset S$ is obtained from some $i$ making an elementary increasing along the $i$-th component if and only if $\eta_{i \mid S_{i}}$ is not injective.

Remark 3.4. Take $S, S_{i}, o, o^{\prime}, o^{\prime \prime}$ as in Example 3.3. Since $\nu(o) \in\left\langle\nu\left(\left\{o^{\prime}, o^{\prime \prime}\right\}\right)\right\rangle$, so $\langle\nu(S)\rangle \subseteq\left\langle\nu\left(S_{i}\right)\right\rangle$, with strict inclusion if and only if $S_{i}$ is linearly independent.

Lemma 3.5. Let $S \subset Y$ be a linearly independent finite subset, $S \neq \emptyset$, such that there exists $i \in\{1, \ldots, k\}$ such that $\left\langle\pi_{i}(S)\right\rangle \subsetneq \mathbb{P}^{n_{i}}$. Then $S$ has a linearly independent elementary increasing in the $i$-th direction.

Proof. Set $s:=\#(S)$. Let $Y^{\prime} \subset Y$ be the multiprojective space with $\mathbb{P}^{n_{j}}$ as its factor for all $j \neq i$ and $\left\langle\pi_{i}(S)\right\rangle$ as its $i$-th factor. By assumption we have $Y^{\prime} \subsetneq Y$. Fix $o \in S$. A general line $L \subset \mathbb{P}^{n_{i}}$ containing $\pi_{i}(o)$ is not contained in $\left\langle\pi_{i}(S)\right\rangle$. Call $Y^{\prime \prime} \subseteq Y$ the multiprojective space with $\mathbb{P}^{n_{j}}$ as its factor for all $j \neq i$ and $\left\langle\pi_{i}(S) \cup L\right\rangle$ as its $i$-th factor. We have $S \subset Y^{\prime} \subsetneq Y^{\prime \prime} \subseteq Y$. Hence $\nu(L) \nsubseteq\left\langle\nu\left(Y^{\prime}\right)\right\rangle$. Fix any $o_{i}^{\prime}, o_{i}^{\prime \prime} \in \pi_{i}(L) \backslash\left\{\pi_{i}(o)\right\}$ such that $o_{i}^{\prime} \neq o_{i}^{\prime \prime}$. Take $o^{\prime}, o^{\prime \prime} \in Y$ with $\pi_{i}\left(o^{\prime}\right)=o_{i}^{\prime}, \pi_{i}\left(o^{\prime \prime}\right)=o_{i}^{\prime \prime}$ and $\pi_{j}\left(o^{\prime}\right)=\pi_{j}\left(o^{\prime \prime}\right)=o_{j}$ for all $j \neq i$.

Remark 3.6. For each $a=\left(a_{1}, \ldots, k\right\}$ set $a[i]:=\eta_{i}^{-1}\left(\eta_{i}(a)\right)$. Note that $a[i] \cong \mathbb{P}^{n_{i}}$ and that $\nu(a[i])$ is an $n_{i}$-dimensional linear space containing $\nu(a)$. Set $\{\{a\}\}:=\cup_{i=1}^{k} a[i]$. Note that $\operatorname{dim}\langle\nu(\{\{a\}\})\rangle=n_{1}+\cdots+n_{k}$. For any finite set $A \subset Y, A \neq \emptyset$, let $A[i]:=\cup_{a \in A} a[i]$ and $\{\{A\}\}:=\cup_{a \in A}\{\{a\}\}$. Now assume that $A$ is linearly independent and $\#(A) \leq r$. Since any two points of a projective space are contained in a line, $A$ has a linearly independent $i$-increasing if and only if $\langle\nu(A)\rangle \subsetneq\langle\nu(A[i])\rangle$; also, $A$ has a linearly independent increasing if and only if $\langle\nu(A)\rangle \subsetneq\langle\nu(\{\{A[i])\}\}\rangle)$. By Lemma 3.5, to prove that $A$ has a linearly independent elementary increasing we may assume that $\left\langle\pi_{i}(A)\right\rangle=\mathbb{P}^{n_{i}}$ for all $i$. Note that if $k=2$ and $\left\langle\pi_{i}(A)\right\rangle=\mathbb{P}^{n_{i}}$ for at least one $i$, then $\langle\nu(A)\rangle=\mathbb{P}^{r}$. 
Remark 3.7. Fix a linearly independent $S \subset Y$ and take $i \in\{1, \ldots, k\}$ and $a \in Y_{i}$. Since 3 collinear points are not linearly independent, we have $\#\left(S \cap \eta_{i}^{-1}(a)\right) \leq 2$. Let $A \subset Y$ be a circuit. Either $\#\left(A \cap \eta_{i}^{-1}(a)\right) \leq 2$ or $A$ is formed by 3 collinear points and so $\mathbb{P}^{1}$ is the minimal multiprojective space containing $A$.

Let $S \subset Y$ be a finite set such that $e(S)>0$. Note that $\#(S) \geq e(S)+2$. A point $o \in S$ is said to be essential for $S$ if $e(S \backslash\{o\})=e(S)-1$. If $o$ is not essential for $S$ we will often say that $o$ is inessential for $S$. Since $e(S)-e\left(S^{\prime}\right) \leq \#(S)-\#\left(S^{\prime}\right)$ for all $S^{\prime} \subset S, o$ is inessential for $S$ if and only if $e(S \backslash\{o\})=e(S)$.

Let $S \subset Y$ be a finite set such that $e(S)>0$. A kernel of $S$ is a minimal subset $S^{\prime} \subseteq S$ such that $e\left(S^{\prime}\right)=e(S)$.

Lemma 3.8. Any finite linearly dependent subset of $Y$ has a unique kernel.

Proof. Take a finite set $S \subset Y$ such that $e(S)>0$. Let $S^{\prime}$ and $S^{\prime \prime}$ be kernels of $S$. Assume $S^{\prime} \neq S^{\prime \prime}$. By the definition of kernels we have $S^{\prime \prime} \nsubseteq S^{\prime}$. Order the points of $S^{\prime \prime}$, say $S^{\prime \prime}=\left\{o_{1}, \ldots, o_{b}\right\}$ so that $o_{b} \notin S^{\prime}$. By the definition of kernel we have

$$
\operatorname{dim}\langle\nu(S)\rangle=\operatorname{dim}\left\langle\nu\left(S^{\prime}\right)\right\rangle+\#\left(S \backslash S^{\prime}\right)
$$

Set $S_{1}:=\left\{o_{1}, \ldots, o_{b-1}\right\} \cup S^{\prime} \supseteq S^{\prime}$ to $S^{\prime}$. Note that (1) gives

$$
\operatorname{dim}\left\langle\nu\left(S_{1}\right)\right\rangle=\operatorname{dim}\left\langle\nu\left(S^{\prime}\right)\right\rangle+b-1-\#\left(S^{\prime} \cap S^{\prime \prime}\right) .
$$

Set $S^{\prime \prime}:=S_{1} \cup\left\{o_{b}\right\}$. Since $e\left(S^{\prime \prime}\right)>0$, we have $\nu\left(o_{b}\right) \in\left\langle\nu\left(\left\{o_{1}, \ldots, o_{b-1}\right\}\right\rangle\right.$ and hence $\nu\left(o_{b}\right) \in\left\langle\nu\left(S_{1}\right)\right\rangle$, contradicting (1).

Lemma 3.9. Let $S \subset Y$ be a finite subset such that $e(S)>0$. The kernel of $S$ is the set of all its essential points; that is, the tail of $S$ is the set of all its inessential points.

Proof. Fix an inessential point $o \in S$ (if any). Let $S^{\prime} \subset S \backslash\{o\}$ be a minimal subset of $S \backslash\{o\}$ with $e\left(S^{\prime}\right)=e(S)$. By Lemma $3.9 S^{\prime}$ is the unique kernel of $S$. Hence the tail of $S$ contains $o$. Thus the tails of $S$ contains all inessential points of $S$. No essential point of $S$ may belong to the tail.

Let $S \subset Y$ be a finite subsets with $e(S)>0$. The tail of $S$ is $S \backslash S^{\prime}$, where $S^{\prime}$ is the kernel of $S$. The tail of $S$ is the set of all inessential points of $S$, while the kernel of $S$ is the set of all its essential points.

Remark 3.10. Let $A \subset \mathbb{P}^{m}$ be a linearly dependent finite subset, that is, a finite subset such that $e(A):=\#(A)-1-\operatorname{dim}\langle A\rangle>0$. We first show different methods to get a subset $B \subseteq A$ with $e(B)=e(A), e\left(B^{\prime}\right)$. Examples show that each of these methods does not give a unique $B$. The first method is increasing the number of points in a subset of $A$. We start with $o, o^{\prime} \in A$ such that $o \neq o^{\prime} ;$ then $e\left(\left\{o, o^{\prime}\right\}\right)=0$.

Consider the following construction: linear projections of a multiprojective space from proper linear subspaces of one of its factors. Fix integers $0 \leq v<n$ and a $v$-dimensional linear subspace $V \subset \mathbb{P}^{n}$. Let $\ell_{V}: \mathbb{P}^{n} \backslash V \rightarrow \mathbb{P}^{n-v-1}$ denote the linear projection from $V$. Take $Y=\mathbb{P}^{n_{1}} \times \cdots \times \mathbb{P}^{n_{k}}$. Fix an integer $i \in\{1, \ldots, k\}$, an integer $v$ such that $0 \leq v<n_{i}$ and a $v$-dimensional linear subspace $V \subset \mathbb{P}^{n_{i}}$. Let $Y^{\prime}$ be the multiprojective space with $k$ factors, with $\mathbb{P}^{n_{h}}$ as its $h$-th factor for all $h \neq i$ and with $V$ as its $i$-th factor. Let $Y^{\prime \prime}$ be the space with $\mathbb{P}^{n_{i}-v-1}$ as its $i$-th factor. The space $Y^{\prime}$ has $k$ non-trivial factors if and only if $v>0$, whereas $Y^{\prime \prime}$ has $k$ non-trivial factors if and only if $v \leq n_{i}-2$. Let $\ell_{V, i}: Y \backslash Y^{\prime} \rightarrow Y^{\prime \prime}$ denote the morphism defined by the formula $\ell_{V, i}\left(a 1, \ldots, a_{k}\right)=\left(b_{1}, \ldots, b_{k}\right)$ with $b_{h}=a_{h}$ for all $h \neq i$ and $b_{i}=\ell_{V}\left(a_{i}\right)$. When $K$ is infinite we use the Zariski topology on $\mathbb{P}^{n}(K)$ and the $K$-points of the Grassmannians.

Remark 3.11. Fix a finite set $S \subset Y$, an integer $i \in\{1, \ldots, s\}$ and an integer $v$ such that $0 \leq v \leq n_{i}-2$. Assume for the moment that $K$ is infinite. Let $V$ be a general (for the Zariski topology) $v$-dimensional linear subspace. Then $\ell_{V, i \mid S}$ is injective. For fixed $k, n_{1}, \ldots, n_{k}$ there is an integer $q_{0}$ such that for all $q \geq 2$ there is a $v$-dimensional linear subspace $V \subset \mathbb{P}^{n_{i}}\left(\mathbb{F}_{q}\right)$ such that $\ell_{V, i \mid S}$ is injective. When $\ell_{V, i \mid S}$ is injective, then $h^{1}\left(Y, \mathcal{I}_{S}(1, \ldots, 1)\right) \leq h^{1}\left(Y^{\prime \prime}, \mathcal{I}_{\ell_{V, i}(S)}(1, \ldots, 1)\right)$. If $Y$ is the minimal multiprojective subspace containing $S$, then $Y^{\prime \prime}$ is the minimal multiprojective space containing $\ell_{V, i}(S)$. 


\section{Rational normal CURVEs inside a SEgre VARIETy}

Fix positive integers $k$ and $n_{i}, 1 \leq i \leq k$. Set $Y:=\mathbb{P}^{n_{1}} \times \cdots \times \mathbb{P}^{n_{k}}$. Let $\mathcal{B}\left(n_{1}, \ldots, n_{k}\right)$ denote the set of all integral curves $D \subset \mathbb{P}^{1}$ such that $D=h\left(\mathbb{P}^{1}\right)$ with $h=\left(h_{1}, \ldots, h_{k}\right): \mathbb{P}^{1} \rightarrow Y$ with $h_{i}: \mathbb{P}^{1} \rightarrow \mathbb{P}^{n_{i}}$ an embedding with $h_{i}\left(\mathbb{P}^{1}\right)$ a rational normal curve of $\mathbb{P}^{n_{i}}$. The set $\mathcal{B}(n)$ of all rational normal curves of $\mathbb{P}^{n}$ is a rational variety of dimension $n(n+3)$. Thus $\mathcal{B}\left(n_{1}, \ldots, n_{k}\right)$ is parametrized by an irreducible variety. For any $D \in \mathcal{B}\left(n_{1}, \ldots, n_{k}\right)$ we have $\operatorname{dim}\langle\nu(D)\rangle=n_{1}+\cdots+n_{k}$ and $\nu(D)$ is a rational normal curve of $\langle\nu(D)\rangle$ of degree $n_{1}+\cdots+n_{k}$. Let $D \subset Y$ be a curve. Then $D \in \mathcal{B}\left(n_{1}, \ldots, n_{k}\right)$ if and only if the following conditions are satisfied:

(a) $D$ is an integral curve;

(b) $\pi_{i \mid D}$ is birational onto its image for all $i=1, \ldots, k$;

(c) $p_{i}(D)$ is a rational normal curve of $\mathbb{P}^{n_{i}}$ for all $i=1, \ldots, k$.

Also, $D \in \mathcal{B}\left(n_{1}, \ldots, n_{k}\right)$ if and only if the following conditions are satisfied:

$\left(\mathrm{a}_{1}\right) D$ is an integral curve;

$\left(\mathrm{b}_{1}\right) \operatorname{deg}(\nu(D))=n_{1}+\cdots+n_{k}$;

$\left(c_{1}\right) Y$ is the minimal multiprojective subspace of $Y$ containing $D$

Remark 4.1. The integer $n_{1}+\cdots+n_{k}$ is the minimal degree of a connected and reduced curve $\nu(D)$, where $D \subset Y$.

Remark 4.2. Fix $D \in \mathcal{B}\left(n_{1}, \ldots, n_{k}\right)$ and any finite subset $S \subset D$. Since $\operatorname{dim}\langle\nu(D)\rangle=n_{1}+\cdots+n_{k}$ and $\nu(D)$ is a rational normal curve of $\langle\nu(D)\rangle$, we have

$$
e(S)=\max \left\{0, \#(S)-n_{1}-\cdots-n_{k}-1\right\} .
$$

Since $\#(L \cap \nu(D)) \leq 1$ for each $L \subset \nu(Y), S$ is minimal.

Remark 4.3. Take $Y:=\left(\mathbb{P}^{1}\right)^{k}$. Let $T \subset Y$ be an integral curve. The multidegree $\left(a_{1}, \ldots, a_{k}\right)$ of $T$ is defined in the following way. If $\pi_{i}(T)$ is a point, then set $a_{i}:=0$. If $\pi_{i}(T)=\mathbb{P}^{1}$ let $a_{i}$ be the degree of the morphism $\pi_{i \mid T}: T \rightarrow \mathbb{P}^{1}$. If $k=3$, we say tridegree instead of multidegree. Note that if $a_{i}>0$ for all $i$, then $T$ is not contained in a proper multiprojective subspace of $Y$. If $a_{i}=1$ for some $i$, then $\pi_{i \mid T}: T \rightarrow \mathbb{P}^{1}$ is a degree 1 morphism between integral curves with the target smooth. By Zariski's Main Theorem $([9]) \pi_{i \mid T}$ is an isomorphism and in particular $T \cong \mathbb{P}^{1}$. Let $\mathcal{B}_{k}$ denote the set of all $T \subset Y$ with multidegree $(1, \ldots, 1)$. We just say that for any $T \in \mathcal{B}_{k}, T \cong \mathbb{P}^{1}$ and each $\pi_{i \mid T}: T \rightarrow \mathbb{P}^{1}$ is an isomorphism. Thus $\mathcal{B}_{k}$, as an algebraic set, is isomorphic to $\operatorname{Aut}\left(\mathbb{P}^{1}\right)^{k}$. Also, $\mathcal{B}_{k}=\mathcal{B}(1, \ldots, 1)$.

Then $D \in \mathcal{B}_{k}$ if and only if the following conditions are satisfied:

$\left(\mathrm{a}_{2}\right) D$ is an integral curve;

$\left(\mathrm{b}_{2}\right) \mathrm{D}$ has multidegree $(1, \ldots, 1)$

Example 4.4. Fix positive integers $e, k$ and $n_{i}, 1 \leq i \leq k$, such that $k \geq 2$. Set $Y=\mathbb{P}^{n_{1}} \times \cdots \times \mathbb{P}^{n_{k}}$ and $m:=\max \left\{n_{1}-1, n_{2}, \ldots, n_{k}\right\}$. Fix a line $L \subseteq \mathbb{P}^{n_{1}}$ and $e+2$ points $a_{1}, \ldots, a_{e+2} \in L$. Fix $o_{i} \in \mathbb{P}^{n_{i}}, 2 \leq i \leq k$. Let $b_{i} \in Y, 1 \leq i \leq e+2$, be the point of $Y$ with $\pi_{1}\left(b_{i}\right)=a_{i}$ and $\pi_{h}\left(b_{i}\right)=o_{h}$ for all $h \in\{2, \ldots, k\}$. Fix any $A \subset Y$ such that $\#(A)=m, \pi_{1}(A) \cup L$ spans $\mathbb{P}^{n_{1}}$ and $\pi_{h}(A)$ spans $\mathbb{P}^{n_{h}}$ for all $h \in\{2, \ldots, k\}$. Set $S:=A \cup\left\{b_{1}, \ldots, b_{e+2}\right\}$. Note that $\#(S)=e+2+m, S$ is not contained in a proper multiprojective subspace of $Y$ and $e(S)=e$.

Proposition 4.5. Fix positive integers $k$ and $n_{i}, 1 \leq i \leq k$, such that $k \geq 2$ and $n_{i} \leq n_{1}$ for all $i$. Let $S \subset Y$ be a finite subset of $Y$ such that $e(S)>0$ and $S$ is not contained in a proper multiprojective subspace of $Y$. Then

$$
\#(S) \geq e(S)+n_{1}+1 .
$$

Proof. Since the proposition is trivial if $k=1$ we may assume $k \geq 2$ and that the proposition is true for multiprojective spaces with smaller dimension. Up to a permutation of the factors of $Y$ we may assume $\mathcal{A}=\{1, \ldots, c\}$. We first consider sets $S$ with $e(S)=1$. Set $s:=\#(S)$. Fix $E \subseteq S$ such that $\#(E)=\min \left\{n_{1}, s\right\}$. Since $h^{0}\left(\mathcal{O}_{Y}\left(\varepsilon_{1}\right)\right)=1$,

Take $H \in\left|\mathcal{O}_{Y^{\prime}}\left(\varepsilon_{1}\right)\right|$ containing at least $m_{1}$ points of $S$. Since $S \nsubseteq H$, Lemma 4.5 gives $h^{1}\left(\mathcal{I}_{S \backslash S \cap H}\left(\hat{\varepsilon}_{1}\right)\right)>0$. Thus $\#(S \backslash S \cap H) \geq 2$. Hence $\#(S) \geq n_{1}+2$. 
Now assume $e(S) \geq 2$. We use induction on the integer $e(S)$. Fix $p \in S$ and set $S^{\prime}:=S \backslash\{p\}$. We have $e(S)-1 \leq e\left(S^{\prime}\right) \leq e(S)$. Assume $e\left(S^{\prime}\right)=e(S)-1$. In this case we have $\left\langle\nu\left(S^{\prime}\right)\right\rangle=\langle\nu(S)\rangle$ and hence $S^{\prime}$ generates $Y$. If $e(S)=e\left(S^{\prime}\right)$ use that the maximal dimension of a factor of the multiprojective space spanned by $S^{\prime}$ is at least $n_{1}-1$.

Proposition 4.6. Let $S \subset Y=\left(\mathbb{P}^{1}\right)^{k}$ be a minimal and non-degenerate set with $e(S)>0$. Then

(a) $\#(S) \geq k+e(S)+1$;

(b) there exists $D \in \mathcal{B}_{k}$ containing $S$ if and only if the $k$ ordered sets $\pi_{i}(S), 1 \leq i \leq s$, are projectively equivalent; that is, there is an ordering $q_{1}, \ldots, q_{s}$ of the points of $S$ and for all $i \neq j$ isomorphisms $h_{i j}: \mathbb{P}^{1} \rightarrow \mathbb{P}^{1}$ such that $h_{i j}\left(\pi_{i}\left(q_{h}\right)\right)=\pi_{j}\left(q_{h}\right)$ for all $h \in\{1, \ldots, s\}$.

Proof. Set $s:=\#(S)$. Since $S$ is minimal, each $\pi_{i \mid S}$ is injective. Assume for the moment $e(S) \geq 2$ and hence $\#(S) \geq e(S)+2$ ). Take any $A \subset S$ such that $\#(A)=\#(S)-e(S)+1$. Since $S$ is minimal and $\#(A) \geq 2, A$ is minimal and spans $Y$. Thus to prove part (a) it is sufficient to prove that $s \geq k+2$ if $e(S)>0$. We use induction on $k$, using in the case $k=1$ the stronger observation that any two points of a line are linearly independent. Assume $k \geq 2$. Fix $o \in S$ and set $\{H\}:=\left|\mathcal{I}_{o}\left(\varepsilon_{k}\right)\right|$. Apply Lemma 2.2 to any partition of $S$ into two proper subsets. Since $S \nsubseteq H$, we have $h^{1}\left(\mathcal{I}_{S \backslash S \cap H}\left(\hat{\varepsilon}_{k}\right)\right)>0$. Hence $\#(S \backslash S \cap H) \geq 2$. Since $S$ is minimal, $\eta_{k \mid S}$ is injective, $\eta_{k}(S)$ is minimal in $Y_{k}$ and $h^{1}\left(\mathcal{I}_{S \backslash S \cap H}\left(\hat{\varepsilon}_{k}\right)\right)=h^{1}\left(Y_{k}, \mathcal{I}_{\eta_{k}(S \backslash S \cap H)}(1, \ldots, 1)\right)$. Thus $\eta_{k}(S \backslash S \cap H)$ is minimal. Since $\#\left(\pi_{i}(S)\right)=s$ for all $i$ and $\#(S \backslash S \cap H)=2, \eta_{k}(S)$ generates $Y_{k}$. The inductive assumption gives $\#(S \backslash S \cap H) \geq k+1$; that is, $s \geq k+2$.

Now we prove part (b). First assume the existence of $D \in \mathcal{B}_{k}$ containing $S$. Write $D=h\left(\mathbb{P}^{1}\right)$ with $h=\left(h_{1}, \ldots, h_{k}\right)$. Each $\pi_{i \mid D}: D \rightarrow \mathbb{P}^{1}$ is an isomorphism. Hence $\pi_{i \mid D} \circ \pi_{j \mid D}^{-1}: \mathbb{P}^{1} \rightarrow \mathbb{P}^{1}$ is an isomorphism sending $\pi_{j}(S)$ onto $\pi_{S}(S)$. Now we assume that all sets $\pi_{i}(S), 1 \leq i \leq s$, are projectively equivalent. We order the points $q_{1}, \ldots, q_{s}$ of $S$. For any $i \geq 2$ let $f_{i}: \mathbb{P}^{1} \rightarrow \mathbb{P}^{1}$ be the isomorphism sending $\pi_{1}\left(q_{x}\right)$ to $\pi_{j}\left(q_{x}\right)$ for all $x \in\{1, \ldots, s\}$. We take the target of $\pi_{1}$ as the domain, $\mathbb{P}^{1}$, of the morphism $h=\left(h_{1}, \ldots, h_{k}\right): \mathbb{P}^{1} \rightarrow Y$ we want to construct. With this condition $h_{1}$ is the identity map. Set $h_{i}:=f_{i}, i=2, \ldots, k$. By construction and the choice of $h_{1}$ as the identity map, we have $h\left(q_{x}\right)=q_{x}$ for all $x \in\{1, \ldots, s\}$. We have $D:=h\left(\mathbb{P}^{1}\right) \in \mathcal{B}_{k}$ and $S \subset D$.

Proposition 4.7. Set $Y:=\mathbb{P}^{n_{1}} \times \cdots \times \mathbb{P}^{n_{k}}, n_{i}>0$ for all $i$, such that $k \geq 2$. Set $m:=$ $\max \left\{n_{1}, \ldots, n_{k}\right\}$. Fix a positive integer $e$. The integer $m+k+e$ is the minimal cardinality of a minimal and non-degenerate set $S \subset Y$ such that $e(S)=e$.

Proof. With no loss of generality we may assume $n_{1}=m$. Fix $Y^{\prime} \subset Y$ with $Y^{\prime}=\left(\mathbb{P}^{1}\right)^{k}$ and take $S^{\prime} \subset S$ with \# $\left(S^{\prime}\right)=k+e+1, S^{\prime}$ non-degenerate and minimal (we may take as $S^{\prime} k+e+1$ points on any $C \in \mathcal{B}_{k}$ ). Then add $m-1$ sufficiently general points of $Y$.

To prove the minimality of the integer $k+e+1$ one can use induction on the integer $m+k$ using linear projections in the single factors $\ell_{\{o\}, i}$ from points of $S$ (Section 3). The starting point of the induction is Proposition 4.6.

Question 4.8. Which are the best lower bounds for \# $(S)$ in Proposition 4.7 if $S$ has no inessential points or it is strongly essential?

\section{Linearly DEPENDENT SUBSETS With LOW CARDinALITY}

Unless otherwise stated $Y=\mathbb{P}^{n_{1}} \times \cdots \times \mathbb{P}^{n_{k}}, n_{i}>0$ for all $i$, and $S \subset Y$ is is linearly dependent and non-degenerate; that is, $S \nsubseteq Y^{\prime}$ for any multiprojective space $Y^{\prime} \subsetneq Y$. Since $\nu$ is an embedding, so $\#(S) \geq 3$.

Remark 5.1. Assume \#(S)=3, that is, assume that $\nu(S)$ spans a line. Since $\nu(Y)$ is cut out by quadrics, we have $\langle\nu(S)\rangle \subseteq \nu(Y)$. Since $S$ is non-degenerate, we have $k=1$ and $n_{1}=1$.

Proposition 5.2. Let $S \subset Y=\prod_{i=1}^{k} \mathbb{P}^{n_{i}}, n_{i}>0$ for all $i$, be a non-degenerate circuit. Assume $\#(S)=4$. Then either $k=1$ and $n_{1}=2$ or $k=2$ and $n_{1}=n_{2}=1$.

Assume $k=2$. In this case $S$ is contained in a unique $D \in\left|\mathcal{O}_{Y}(1,1)\right|$.

(a) Assume that $D$ is reducible, say $D=D_{1} \cup D_{2}$ with $D_{1} \in\left|\mathcal{O}_{Y}(1,0)\right|$ and $D_{2} \in\left|\mathcal{O}_{Y}(0,1)\right|$, then $\#\left(S \cap D_{1}\right)=\#\left(S \cap D_{2}\right)=2$ and $D_{1} \cap D_{2} \cap S=\emptyset$. For any choice of $A \subset S$ with $\#\left(A \cap D_{1}\right)=\#\left(A \cap D_{2}\right)=1$, the set $\langle\nu(A)\rangle \cap\langle\nu(S \backslash A)\rangle$ is a single point, q. We have $r_{X}(q)=2$ and $A, S \backslash A \in \mathcal{S}(Y, q)$. 
(b) Assume that $D$ is irreducible. For any choice of a set $A \subset S$ such that \# $(A)=2$ the set $\langle\nu(A)\rangle \cap\langle\nu(S \backslash A)\rangle$ is a single point, $q$. Then $r_{X}(q)=2$ and $A, S \backslash A \in \mathcal{S}(Y, q)$.

Proof. If $k=1$, then $S$ is formed by 4 coplanar points, no 3 of them collinear. The minimality assumption of $Y$ gives $n_{1}=2$.

From now on we assume $k \geq 2$. Take $A \subset S$ such that $\#(A)=2$ and set $B:=S \backslash A$. Since $h^{1}\left(\mathcal{I}_{S}(1, \ldots, 1)\right)>0$ and $h^{1}\left(\mathcal{I}_{S^{\prime}}(1, \ldots, 1)\right)=0$ for all $S^{\prime} \subsetneq S$, the set $\langle\nu(A)\rangle \cap\langle\nu(B)\rangle$ is a single point, $q$. Since $\nu(S)$ is a circuit, we have $q \notin \nu(S)$. If $q \notin \nu(Y)$, then $A$ shows that $r_{X}(q)=2$.

(a) Assume $q \in \nu(Y)$, say $q=\nu(o)$. Since $S$ is a circuit, we saw that $o \notin S$.

Since $\#(\langle\nu(A)\rangle \cap \nu(Y)) \geq 3$, \# $(\langle\nu(B)\rangle \cap \nu(Y)) \geq 3$ and $\nu(Y)$ is cut out by quadrics, we have $\langle\nu(A)\rangle \cup\langle\nu(B)\rangle \subset \nu(Y)$, i.e., there are integers $i, j \in\{1, \ldots, k\}$ such that

$$
\#\left(\pi_{h}(A)\right)=\#\left(\pi_{m}(B)\right)=1
$$

for all $h \neq i$ and all $m \neq j$. Since $\nu(S) \subset\langle\nu(A)\rangle \cup\langle\nu(B)\rangle$ and $\nu(o) \in\langle\nu(A)\rangle \cap\langle\nu(B)\rangle$, the minimality of $Y$ gives $k=2, n_{1}=n_{2}=1$ and that we are in case (a).

(b) From now on we assume $q \notin \nu(Y)$.

Claim 1: Each $\pi_{i \mid S}: S \rightarrow \mathbb{P}^{n_{i}}$ is injective.

Proof: Assume that some $\pi_{i \mid S}$ is not injective, say $\pi_{1 \mid S}$ is not injective. Thus there is $A^{\prime} \subset S$ such that $\#\left(A^{\prime}\right)=2$ and $\#\left(\pi_{1}\left(A^{\prime}\right)\right)=1$. Set $B^{\prime}:=S \backslash A^{\prime}$. We see that $A^{\prime}, B^{\prime}$ are as in case (a) and in particular $k=2$ and $n_{1}=n_{2}=2$ and $S=A^{\prime} \cup B^{\prime} \subset D_{1} \cup D_{2}$.

Claim 2: We have $n_{i}=1$ for all $i$.

Proof: Assume the existence of $i \in\{1, \ldots, k\}$ such that $n_{i} \geq 2$. Since $h^{0}\left(\mathcal{O}_{Y}\left(\varepsilon_{i}\right)\right) \geq 3$, there is $H \in\left|\mathcal{O}_{Y}\left(\varepsilon_{i}\right)\right|$ containing $A$. The minimality of $Y$ and the inclusion $A \subset H$ implies $B \nsubseteq H$, i.e., $B \backslash B \cap H \neq A \backslash A \cap H$. Lemma 2.2 implies $h^{1}\left(\mathcal{I}_{B \backslash B \cap H}\left(\hat{\varepsilon}_{i}\right)\right)>0$. Since $\mathcal{O}_{Y}\left(\hat{\varepsilon}_{i}\right)$ is a globally generated line bundle, we get $\#(B \backslash B \cap H)>1$. Thus $B \cap H=\emptyset$. Since any Segre embedding is an embedding, we get $\#\left(\pi_{h}(B)\right)=2$ for all $h \neq i$, contradicting Claim 1 because $k \geq 2$.

Claim 3: We have $k=2$ and $n_{1}=n_{2}=1$.

Proof: By Claim 2 it is sufficient to prove that $k=2$. Assume $k \geq 3$. Since $h^{0}\left(\mathcal{O}_{Y}\left(\varepsilon_{1}\right)\right)=2$, there are $H_{i} \in\left|\mathcal{O}_{Y}\left(\varepsilon_{i}\right)\right|, i=1,2$, such that $A \subset H_{1} \cup H_{2}$. Since $k \geq 3$, as in the proof of Claim 2 we get that either $B \subset H_{1} \cup H_{2}$ or $B \cap\left(H_{1} \cup H_{2}\right)=0$ and $\#\left(\pi_{i}(B)\right)=1$ for all $i \geq 3$. The latter possibility is excluded by Claim 1. Thus $S \subset H_{1} \cup H_{2}$. Hence there is $i \in\{1,2\}$ such that $\#\left(H_{i} \cap S\right) \geq 2$; that is, $\pi_{i \mid S}$ is not injective, contradicting Claim 1.

Remark 5.3. In case (b) of Proposition 5.2 $S$ is minimal, while $S$ in case (a) is not minimal.

Case (a) of Proposition 5.2 may be generalized to the following examples of circuits in $\mathbb{P}^{n_{1}} \times \mathbb{P}^{n_{2}}$.

Example 5.4. Take $k=2$, that is, $Y=\mathbb{P}^{n_{1}} \times \mathbb{P}^{n_{2}}$. Fix $o=\left(o_{1}, o_{2}\right) \in Y$ and set $D_{i}:=\pi_{i}^{-1}\left(o_{i}\right)$, $i=1,2$. We have $D_{i} \in\left|\mathcal{O}_{Y}\left(\varepsilon_{i}\right)\right|$. Fix $S_{i} \in D_{i} \backslash\{o\}$ such that $\#\left(S_{i}\right)=n_{3-i}+1$ and $\nu\left(S_{i}\right)$ spans the linear space $\nu\left(D_{i}\right)$. Set $S:=S_{1} \cup S_{2}$. Since $o \notin S_{1} \cup S_{2}$, we have $\#(S)=n_{1}+n_{2}+2$. It may be checked that $S$ is a circuit. We get in this way an irreducible and rational family $T$ of circuits with $\operatorname{dim} T=n_{1}^{2}+n_{2}^{2}+2 n_{1}+2 n_{2}+2$.

Remark 5.5. Let $S \subset Y=\mathbb{P}^{n_{1}} \times \cdots \times \mathbb{P}^{n_{k}}$ be a non-degenerate circuit with cardinality 5 .

(a) Assume $k=1$ and $n_{1}=3$. In this case we may take as $S$ any set with cardinality 5 such that all its proper subsets are linearly independent.

(b) Assume $k=2$ and $n_{1}=n_{2}=1$. Since $r=3$, in this case we may take as $S$ any set with cardinality 5 such that all its proper subsets are linearly independent.

Proposition 5.6. Take $S \subset Y$ with $\#(S)=5$ and $e(S) \geq 2$. Let $A$ be the kernel of $S$ and let $Y^{\prime}=\mathbb{P}^{m_{1}} \times \cdots \times \mathbb{P}^{m_{s}}, s \geq 1$, be the minimal multiprojective space containing $A$. Then $A, e(S)$ and $Y^{\prime}$ are in the following list and all the numerical values in the list are realized by some $S$ :

(1) $e(S)=3, A=S, s=1$ and $m_{s}=1$;

(2) $e(S)=2, A=S, s=1$ and $m_{s}=2$;

(3) $e(S)=2, A=S, s=2$ and $m_{1}=m_{2}=1$;

(4) $e(S)=2, \#(A)=4, s=1$ and $m_{s}=1$.

Proof. We have $e(A)=e(S)$. Since $\nu(Y)$ is cut out by quadrics, each line $L \subset \mathbb{P}^{r}$ containing at least 3 points of $\nu(Y)$ is contained in $\nu(Y)$ and hence $L=\nu(J)$ for some line $J$ in one of the factors of $Y$. 
First assume $\#(A) \leq 4$. Since $e(A) \geq 2$ and $\nu$ is an embedding, we see that $\#(A)=4, e(S)=2$, $s=1$ and $m_{1}=1$.

From now on we assume $\#(A)=5$, i.e., $A=S$.

Since $\nu$ is an embedding, we see that $e(A) \leq 3$ and that $e(A)=3$ if and only if $s=1$ and $m_{1}=1$. Now assume $e(A)=2$. In this case $\langle\nu(A)\rangle$ is a plane $\Pi$ containing at least 5 distinct points of $\nu(A)$. The plane $\Pi$ is contained in $\nu(Y)$ if and only if $s=1$ and $m_{1}=2$. Now assume that $\Pi$ is not contained in $\nu(Y)$. Thus $s \geq 2$. Since $\nu(Y)$ is cut out by quadrics, $\nu(A) \subset \Pi$, and $\#(A)=5$, $\Pi \cap \nu(Y)$ is a conic, $T$. Since $e(A)=2$ and $A$ is a finite set, $T$ is not a double line. Taking suitable $E \subset T$ with $\#(E)=4$ and applying Proposition 5.2 we get $s=2$ and $m_{1}=m_{2}=1$.

Example 5.7. Take $Y=\mathbb{P}^{2} \times \mathbb{P}^{1}$. Let $S \subset Y$ be a non-degenerate circuit with $\#(S)=5$. Here we describe the elements of $D \in\left|\mathcal{O}_{Y}(1,1)\right|$ and the intersections of two of them containing $S$. Here $\operatorname{deg}(\nu(Y))=3$ : by the distributive law the intersection number $\mathcal{O}_{Y}(1,1) \cdot \mathcal{O}_{Y}(1,1) \cdot \mathcal{O}_{Y}(1,1)$ is 3 times the integer $\mathcal{O}_{Y}(1,0) \cdot \mathcal{O}_{Y}(1,0) \cdot \mathcal{O}_{Y}(1,0)=1$. Since $S$ is a circuit, $\#(S)=5$ and $r=5$, so $h^{0}\left(\mathcal{I}_{S}(1,1)\right)=2$.

Claim 1: The base locus $B$ of $\left|\mathcal{I}_{S}(1,1)\right|$ contains no effective divisor.

Proof: Assume that $D$ is an effective divisor contained in $B$. Since $h^{0}\left(\mathcal{I}_{S}(1,1)\right)>1$, we have $\left.D \in \mid \mathcal{O}_{Y} \varepsilon_{i}\right) \mid$ for some $i=1,2$. By assumption we have $h^{0}\left(\mathcal{I}_{S \backslash S \cap D}\left(\hat{\varepsilon}_{i}\right)\right)=h^{0}\left(\mathcal{I}_{S}(1,1)\right)=2$. Since $D$ is a multiprojective space, we have $i=2$ and $\#(S \backslash S \cap D)=1$. Lemma 2.2 gives $h^{1}\left(\mathcal{I}_{S \backslash S \cap D}(1,0)\right)>0$, a contradiction.

By Claim 1, $S$ is contained in a unique complete intersection of two elements of $\left|\mathcal{O}_{Y}(1,1)\right|$.

Suppose $C$ is the complete intersection of two elements of $\left|\mathcal{O}_{Y}(1,1)\right|$ (we allow the case in which $C$ is reducible or with multiple components). We know that $\operatorname{deg}(C)=\operatorname{deg}(\nu(Y))=3$. Since $h^{1}\left(\mathcal{O}_{Y}(-2,-2)\right)=0$ (Künneth), a standard exact sequence gives $h^{0}\left(\mathcal{O}_{C}\right)=1$. Thus $C$ is connected. Since $C$ is the complete intersection of 2 ample divisors, we have $\operatorname{deg}\left(\mathcal{O}_{C}(0,1)\right)=1$ and $\left.\operatorname{deg}\left(\mathcal{O}_{C}(1,0)\right)=\mathcal{O}_{Y}(1,0) \cdot \mathcal{O}_{Y}(1,1) \cdot \mathcal{O}_{Y}(1,1)\right)=2$, where the second term is the intersection product. Since $\omega_{Y} \cong \mathcal{O}_{Y}(-3,-2)$, the adjunction formula gives $\omega_{C} \cong \mathcal{O}_{Y}(-1,0)$. Thus its irreducible components $T$ of $D$ with $\pi_{1}(T)$ not a point is a smooth rational curve of degree $\leq 2$, while all irreducible components of $T$ of $C$ with $\pi_{1}(T)$ a point has arithmetic genus 1 , hence no such $T$ exists.

Now suppose that $S \subset C$. Since $h^{0}\left(\mathcal{I}_{S}(1,1)\right)=2=h^{0}\left(\mathcal{I}_{C}(1,1)\right)$, so $\langle\nu(S)\rangle=\langle\nu(C)\rangle \cong \mathbb{P}^{3}$. If $C$ is irreducible, then it a smooth rational normal curve of $\mathbb{P}^{3}$. In this case any 5 points of $C$ forms a circuit. Of course, the general complete intersection of two elements of $\left|\mathcal{O}_{Y}(1,1)\right|$ is irreducible. In this way we get an irreducible family of dimension 13 of circuits. Now assume that $C$ is not irreducible. Since it is connected and $S \subset C_{\text {red }}$, we get that $C$ has only multiplicity one components, so it is either a connected union of 3 lines with arithmetic genus 0 or a union of a smooth conic and a line meeting exactly at one point and quasi-transversally. Since $\nu(S)$ is a circuit, each line contained in $\nu(C)$ contains at most 2 points of $\nu(S)$ and each conic contained in $\nu(C)$ contains at most 3 points of $S$. Thus if $C=T_{1} \cup L_{1}$ with $\nu\left(T_{1}\right)$ a smooth conic, we have $S \cap T_{1} \cap L_{1}=\emptyset$, \# $\left(S \cap T_{1}\right)=3$ and $\#\left(S \cap L_{1}\right)=2$. Conversely any $S \subset T_{1} \cup L_{1}$ with these properties gives a circuit. The smooth conics, $T$, contained in $Y$ are of two types: either $\pi_{2}(T)$ is a point and $\pi_{1}(T)$ is a conic or $\pi_{2}(T)=\mathbb{P}^{1}$ and $\pi_{1}(T)$ is a line; equivalently the minimal Segre variety containing $T$ is isomorphic to $\left.\mathbb{P}^{1} \times \mathbb{P}^{1}\right)$. Now take $C=L_{1} \cup L_{2} \cup L_{3}$ with $\nu\left(L_{i}\right)$ a line for all $i$. We have $\#\left(S \cap L_{i}\right) \leq 2$ for all $i$. For any $1 \leq i<j \leq 3$ such that $L_{i} \cap L_{j} \neq \emptyset$ we have $\#\left(S \cap L_{i} \cup L_{j}\right) \leq 3$. Conversely any $S$ satisfying all these inequalities gives a circuit.

Lemma 5.8. Let $\Sigma$ be the set of all non-degenerate circuits $S \subset Y:=\mathbb{P}^{1} \times \mathbb{P}^{1} \times \mathbb{P}^{1}$ such that $\#(S)=5$. Let $\mathcal{B}$ be the set of all integral curves $C \subset Y$ of tridegree $(1,1,1)$.

(a) $\Sigma$ is non-empty, irreducible, $\operatorname{dim} \Sigma=11$ and, if $K$ is an algebraically closed field with characteristic $0, \Sigma$ is rationally connected.

(b) $\mathcal{B}$ is irreducible and rational, $\operatorname{dim} \mathcal{B}=6$. For any $C \in \mathcal{B}$ we have $\operatorname{dim}\langle\nu(C)\rangle=3$ and the curve $\nu(C)$ is a rational normal curve of $\langle\nu(C)\rangle$.

(c) Each $S \in \Sigma$ is contained in a unique $C \in \mathcal{B}$.

(d) For any $C \in \mathcal{B}$ any $S \subset C$ with $\#(S)=5$ is an element of $\Sigma$. 
Proof. By Remark $4.3, \mathcal{B}$ is parametrized by the set of all triples $\left(h_{1}, h_{2}, h_{3}\right) \in \operatorname{Aut}\left(\mathbb{P}^{1}\right)^{3}$; we may take as $h_{1}$ the identity map $\mathbb{P}^{1} \rightarrow \mathbb{P}^{1}$. Thus $\mathcal{B}$ is irreducible, rational and of dimension 6 . Hence part (b) is true.

(a) Fix $C \in \mathcal{B}$. Since $C$ is irreducible and with tridegree $(1,1,1)$, it is smooth and rational, $\operatorname{deg}(\nu(C))=3$ and $C$ is not contained in a proper multiprojective subspace of $Y$. Since $\nu(C)$ is smooth and rational and $\operatorname{deg}(\nu(C))=3$, we have $\operatorname{dim}\langle\nu(C)\rangle=3$. Thus $\nu(C)$ is a rational normal curve of $\langle\nu(C)\rangle$. Hence any $A \subset Y$ such that $\#(A)=5$ and $\nu(A) \subset \nu(C)$ is a circuit. To conclude the proof of part (d), it is sufficient to prove that $A$ is not contained in a proper multiprojective subspace of $Y$. We prove that no $B \subset C$ with $\#(B)=2$ is contained in a proper multiprojective subspace of $Y$. Take $B \subset C$ such that $\#(B)=2$. Since $C$ has multidegree $(1,1,1)$ each $\pi_{i \mid C}$ is injective. Thus $\#\left(\pi_{i}(B)\right)=2$ for all $i$; that is, there is no $D \in\left|\mathcal{O}_{Y}\left(\varepsilon_{i}\right)\right|$ containing $B$. If we prove part (c) of the lemma, then part (a) would follow, except for the rational connectedness of $\Sigma$. However, this follows immediately from [8, Corollary 1.3].

(b) Fix $S \in \Sigma$. We have $\operatorname{dim}\langle\nu(S)\rangle=3$. Next, $\#(L \cap \nu(S)) \leq 2$ for any line $L \subset \mathbb{P}^{7}$ and $\#(C \cap \nu(S)) \leq 3$ for any plane curve $C \subset \mathbb{P}^{7}$. Take any $E \subset S$ such that \#(E)=2 and set $F:=S \backslash E$. Since $S$ is a circuit, $\langle\nu(E)\rangle \cap\langle\nu(F)\rangle$ is a single point $q$, and $q \notin\langle\nu(G)\rangle$ for any $G \subsetneq A$ and any $G \subsetneq F$. Fix $i \in\{1,2,3\}$. Since $\operatorname{dim}\left|\mathcal{O}_{Y}\left(\hat{\varepsilon}_{i}\right)\right|=3$, there is $D \in\left|\mathcal{I}_{B}\left(\hat{\varepsilon}_{i}\right)\right|$. Lemma 2.2 gives that either $S \subset D$ or $h^{1}\left(\mathcal{I}_{S \backslash S \cap D}\left(\varepsilon_{i}\right)\right)>0$. If $h^{1}\left(\mathcal{I}_{S \backslash S \cap D}\left(\varepsilon_{i}\right)\right)>0$, then $S \backslash S \cap D=E$ and $\#\left(\pi_{h}(E)\right)=1$ for all $h \in\{1,2,3\} \backslash\{i\}$; that is, \#( $\left.\eta_{i}(E)\right)=1$.

Fix $i \in\{1,2,3\}$ and $A \subset S$ such that $\#(A)=\#\left(\eta_{i}(A)\right)=2$. Set $B:=S \backslash A$. It has just been shown that any $D_{i} \in\left|\mathcal{O}_{Y}\left(\hat{\varepsilon}_{i}\right)\right|$ containing $B$ contains $S$. Since $\left.\#(L \cap \nu(S)\rangle\right) \leq 2$ for any line $L \subset \mathbb{P}^{7}$, we get $\#\left(\eta_{i}(S)\right) \geq 2$ for all $i=1,2,3$. Thus there are $D_{i} \in\left|\mathcal{O}_{Y}\left(\hat{\varepsilon}_{i}\right)\right|, i=1,2,3$ such that $S \subseteq D_{1} \cap D_{2} \cap D_{3}$. Since $h^{0}\left(\mathcal{O}_{Y}\left(\varepsilon_{i}\right)=2\right.$, a standard exact sequence gives $\operatorname{dim}\left\langle\nu\left(D_{i}\right)\right\rangle=5$. Since $\left\langle\nu\left(D_{i}\right) \cup\left\langle\nu\left(D_{j}\right\rangle=\mathbb{P}^{7}\right.\right.$ for all $i \neq j$, the Grassmann formula gives that $\operatorname{dim}\left\langle\nu\left(D_{i}\right)\right\rangle \cap\left\langle\nu\left(D_{j}\right)\right\rangle$. Thus for any $G \in \Sigma$ contained in $D_{i} \cap J$ we have $\left.\langle\nu(G)\rangle=\left\langle\nu\left(D_{i}\right)\right\rangle \cap\left\langle\nu\left(D_{j}\right)\right)\right\rangle$. Assume for the moment that $D_{1}$ is reducible, say $D_{1}=D^{\prime} \cup D^{\prime \prime}$ with $D^{\prime} \in\left|\mathcal{O}_{Y}\left(\varepsilon_{2}\right)\right|$ and $D^{\prime \prime} \in\left|\mathcal{O}_{Y}\left(\varepsilon_{3}\right)\right|$. Take any $M \in\left|\mathcal{O}_{Y}\left(\varepsilon_{2}\right)\right|$ containing no irreducible component of $D_{1}$.

Claim 1: There is no line $L \subset \nu(Y)$ such that $\#(L \cap \nu(S))=2$.

Proof: Assume that $L$ exists and take $A \subset S$ such that $\#(A)=2$ and $\nu(A) \subset L$. By the structure of lines of $\nu(Y)$ there is $i \in\{1,2,3\}$ such that $\#\left(\pi_{h}(A)\right)=1$ for all $h \in\{1,2,3\} \backslash\{i\}$, i.e., $\#\left(\eta_{i}(A)\right)=1$. Set $\{M\}:=\left|\mathcal{I}_{A}\left(\varepsilon_{i}\right)\right|$. Since $M$ is a multiprojective space, we have $S \nsubseteq M$. Thus Lemma 2.2 gives $h^{1}\left(\mathcal{I}_{S \backslash S \cap M}\left(\hat{\varepsilon}_{i}\right)\right)>0$. Thus one of the following cases occur:

(1) there are $u, v \in S \backslash S \cap M$ such that $u \neq v$ and $\eta_{i}(u)=\eta_{i}(v)$;

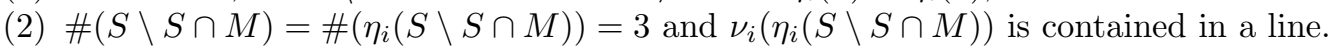

First assume the existence of $u, v$. We get the existence of a line $R \subset \nu(Y)$ such that $\{u, v\} \subset R$ and $R \cap L=\emptyset$. Thus $\operatorname{dim}\langle L \cup R\rangle=3$. Since $L$ and $R$ are generated by the points of $\nu(S)$ contained in them, we get $\nu(S) \subset\langle L \cup R\rangle$. Set $\{o\}:=S \backslash(A \cup\{u, v\})$. Since any line of $\mathbb{P}^{7}$ contains at most 2 points of $\nu(S)$, we get $\nu(o) \in\langle L \cup R\rangle \backslash(L \cup R)$. Thus there is a unique line $J \subset\langle L \cup R\rangle$ such that $J \cap L \neq \emptyset, R \cap J \neq \emptyset$ and $\nu(o) \in J$. Since $\nu(Y)$ is cut out by quadrics and $\{o\} \cup L \cup R \subset \nu(Y)$, we get $J \subset \nu(Y)$. Since $\langle L \cup R\rangle \cap \nu(Y)$ is cut out by quadrics and $\nu(Y)$ contains no plane, we get that $\langle L \cup R\rangle \cap \nu(Y)$ is a quadric surface $Q$.

Subclaim: $Q=\nu(Y))$ for some multiprojective subspace $Y^{\prime} \subsetneq Y$.

Proof: Since the irreducible quadric surface $Q$ contains the lines $R$ and $L$ such that $R \cap L=\emptyset$, we have $Q \cong \mathbb{P}^{1} \times \mathbb{P}^{1}$ and all elements of the two rulings of $Q$ are embedded as lines. The structure of linear spaces contained in Segre varieties gives $Q=\nu\left(Y^{\prime}\right)$ for some 2-dimensional multiprojective subspace $Y^{\prime} \subset Y$.

Since $S \subset\langle L \cup R\rangle \cap \nu(Y)=Q$, the subclaim gives a contradiction.

Now assume \# $(S \backslash S \cap M)=\#\left(\eta_{i}(S \backslash S \cap M)\right)=3$ and that $\nu_{i}\left(\eta_{i}(S \backslash S \cap M)\right)$ is contained in a line. Thus there is $j \in\{1,2,3\} \backslash\{i\}$ such that $\#\left(\pi_{j}\left(\eta_{i}(S \backslash S \cap M)\right)\right)=1$. Since $j \neq i$, we have $\pi_{j}\left(\left(\eta_{i}(S \backslash S \cap M)\right)\right)=\pi_{j}(S \backslash S \cap M)$. Thus there is $W \in\left|\mathcal{O}_{Y}\left(\varepsilon_{j}\right)\right|$ such $S \backslash S \cap M$. Since $W$ is a multiprojective space, we have $S \nsubseteq W$. Thus Lemma 2.2 gives $h^{1}\left(\mathcal{I}_{S \backslash S \cap W}\left(\hat{\varepsilon}_{j}\right)\right)>0$. Since $\mathcal{O}_{Y}\left(\hat{\varepsilon}_{j}\right)$ is globally generated and $S \backslash S \cap W \subseteq A$, we get $S \backslash S \cap W=A$. Since $j \neq i$, so \# $\left(\eta_{j}(A)\right)=2$. Thus $h^{1}\left(\mathcal{I}_{A}\left(\hat{\varepsilon}_{j}\right)\right)=0$, a contradiction.

(c) In step (b), it was shown that $S \subseteq D_{1} \cap D_{2} \cap D_{j}$ for some $D_{i} \in\left|\mathcal{O}_{Y}\left(\hat{\varepsilon}_{i}\right)\right|$ and that $\langle\nu(S)\rangle=\left\langle\nu\left(D_{i}\right)\right\rangle \cap\left\langle\nu\left(D_{j}\right)\right\rangle$. Now we assume that both $D_{1}$ and $D_{2}$ are reducible and that they 
have a common irreducible component. Write $D_{1}=D^{\prime} \cup D^{\prime \prime}$ with $D^{\prime} \in\left|\mathcal{O}_{Y}\left(\varepsilon_{3}\right)\right|$ and $D^{\prime \prime} \in\left|\mathcal{O}_{Y}\left(\varepsilon_{2}\right)\right|$. We see that $D_{1}$ and $D_{2}$ have $D^{\prime}$ as their common component, say $D_{2}=D^{\prime} \cup M$ with $M \in\left|\mathcal{O}_{Y}\left(\varepsilon_{1}\right)\right|$. The curve $\nu\left(D^{\prime \prime} \cap M\right)$ is a line. Since $\operatorname{dim}\left\langle\nu\left(D^{\prime}\right)\right\rangle=3$, we have $\left\langle\nu\left(D_{1}\right)\right\rangle \cap\left\langle\nu\left(D_{2}\right)\right\rangle=\left\langle\nu\left(D^{\prime}\right)\right\rangle$. Since $\mu(Y)$ is cut out by quadrics and contains no $\mathbb{P}^{3}$, so $M \cap D^{\prime \prime} \subset D^{\prime}$. Thus $S \subset D^{\prime}$, a contradiction.

In the same way we exclude the existence of a surface contained in $D_{i} \cap D_{j}$ for any $i \neq j$.

Now assume that $D_{1}=D^{\prime} \cup D^{\prime \prime}$ is reducible, $D_{2}=M^{\prime} \cup M^{\prime \prime}$ is reducible, but that $D_{1}$ and $D_{2}$ have no common irreducible component. Then $\nu\left(D_{1} \cap D_{2}\right)$ is a union of 4 lines. Since $S \subset D_{1} \cap D_{2}$, Claim 1 gives a contradiction. Thus at most one among $D_{1}, D_{2}$ and $D_{3}$ is reducible. Assume that $D_{1}$ is reducible, say $D_{1}=D^{\prime} \cup D^{\prime \prime}$ with $D^{\prime} \in\left|\mathcal{O}_{Y}\left(\varepsilon_{3}\right)\right|$ and $D^{\prime \prime} \in\left|\mathcal{O}_{Y}\left(\varepsilon_{2}\right)\right|$. The curve $\nu\left(D_{2} \cap D^{\prime \prime}\right)$ is a conic, perhaps reducible, and hence it contains at most 3 points of $\nu(S)$. The curve $\nu\left(D_{2} \cap D^{\prime}\right)$ is a line. Since $S \subset D_{1} \cap D_{2}$, Claim 1 gives a contradiction. Thus each $D_{i}, 1 \leq i \leq 3$, is irreducible.

(d) By part (c), $S \subseteq D_{1} \cap D_{2} \cap D_{3}$ with $D_{i} \in\left|\mathcal{O}_{Y}\left(\hat{\varepsilon}_{i}\right)\right|$ and each $D_{i}$ irreducible. Thus $T:=D_{1} \cap D_{2}$ has pure dimension 1 . We have $\mathcal{O}_{Y}(1,1,1) \cdot \mathcal{O}_{Y}(0,1,1) \cdot \mathcal{O}_{Y}(1,0,1)=3$, the intersection number; that is, the curve $\nu(T)$ has degree 3. Since $S \subset T$ and no line contains two points of $\nu(S)$ (Claim 1), $T$ must be irreducible. Since $S \subset T$, no proper multiprojective subspace of $Y$ contains $T$. Thus $T$ has multidegree $\left(a_{1}, a_{2}, a_{3}\right)$ with $a_{i}>0$ for all $i$. Since $\operatorname{deg}(T)=a_{1}+a_{2}+a_{3}$, we get $a_{i}=1$ for all $i$, that is, $T \in \mathcal{B}$. Fix $C, C^{\prime} \in \mathcal{B}$ such that $C \neq C^{\prime}$ and assume $S \subseteq C \cap C^{\prime}$. Then, $\langle\nu(C)\rangle=\langle\nu(S)\rangle=\left\langle\nu\left(C^{\prime}\right)\right\rangle$. Hence $\langle\langle\nu(S)\rangle \cap \nu(Y)$ contains two different rational normal curves of $\mathbb{P}^{3}$ with 5 common points. Such a reducible curve $\nu(C) \cup \nu\left(C^{\prime}\right)$ is contained in a unique quadric surface $Q^{\prime}$, and $Q^{\prime}$ is integral. Since $\nu(Y)$ is cut out by quadrics, there is a an integral surface $G \subset X$ such that $\nu(G)=Q^{\prime}$. Since $\operatorname{deg}(G)=2$, so $G \in\left|\mathcal{O}_{Y}\left(\varepsilon_{i}\right)\right|$ for some $i$. Since $G$ is a multiprojective space and $S \subset G$, a contradiction is obtained.

Proof of Theorem 1.1: The cases $k=1$ and $k=2$ with $n_{1}=n_{2}=1$ are straightforward. Thus it may be assumed that $k \geq 2$ and $n_{1}+\cdots+n_{k} \geq 3$.

(a) Assume $k=2, n_{1}=2$ and $n_{2}=1$. Thus $r=5$. All $S \in \Sigma$ are described in Example 5.7. The same proof works if $k=2, n_{1}=1$ and $n_{2}=2$.

(b) Assume $k=2$ and $n_{1}=n_{2}=2$. Fix $a, a^{\prime} \in S, a \neq a^{\prime}$ and take $H \in\left|\mathcal{O}_{Y}(1,0)\right|$ containing $\left\{a, a^{\prime}\right\}$. Since $S \nsubseteq H$, Lemma 2.2 gives $h^{1}\left(\mathcal{I}_{S \backslash S \cap H}(0,1)\right)>0$. Thus either there are $b, b^{\prime} \in S \backslash S \cap H$ such that $b \neq b^{\prime}$ and $\pi_{2}(b)=\pi_{2}\left(b^{\prime}\right)$ or $\#(S \backslash S \cap H)=3$ and $\pi_{2}(S \backslash S \cap H)$ is contained in a line.

First assume the existence of $b, b^{\prime}$. Write $S=\left\{b, b^{\prime}, u, v, w\right\}$. Then there exists $D \in\left|\mathcal{O}_{Y}(0,1)\right|$ containing $\left\{b, b^{\prime}, u\right\}$. Since $S \nsubseteq D$, Lemma 2.2 gives $h^{1}\left(\mathcal{I}_{S \backslash S \cap D}(1,0)\right)>0$. Thus $S \backslash S \cap D=\{v, w\}$ and $\pi_{2}(v)=\pi_{2}(w)$. Taking $w$ instead of $u$, then $\pi_{2}(v)=\pi_{2}(v)=\pi_{2}\left(w_{2}\right)$. Hence $U \in\left|\mathcal{O}_{Y}(0,1)\right|$ containing at least 4 points of $S$. Since $U$ is a multiprojective space, we have $S \nsubseteq U$. Lemma 2.2 gives $h^{1}\left(\mathcal{I}_{S \backslash S \cap U}(1,0)\right)>0$. Since $\#(S \backslash S \cap U)=1$, we get a contradiction.

Now assume that $\pi_{2}\left(S \backslash\left\{a, a^{\prime}\right\}\right)$ is contained in a line. Thus there is $M \in\left|\mathcal{O}_{Y}(0,1)\right|$ containing $S \backslash\left\{a, a^{\prime}\right\}$. Since $S \nsubseteq M$, Lemma 2.2 gives $h^{1}\left(\mathcal{I}_{S \backslash S \cap M}(1,0)\right)>0$. Since $S \backslash S \cap M \subseteq\left\{a, a^{\prime}\right\}$, we get $\pi_{1}(a)=\pi_{1}\left(a^{\prime}\right)$. We conclude as we did with $\left\{b, b^{\prime}\right\}$ using the other factor of $\mathbb{P}^{2} \times \mathbb{P}^{2}$.

(c) Assume $k=2, n_{1}=3$ and $n_{2}=1$. Take $H \in\left|\mathcal{O}_{Y}(1,0)\right|$ containing $B$. Since $H$ is a multiprojective space, we have $S \nsubseteq H$. Thus Lemma 2.2 gives $h^{1}\left(\mathcal{I}_{A \backslash A \cap H}(0,1)\right)>0$. Since $\mathcal{O}_{\mathbb{P}^{1}}(1)$ is very ample and $\#(A \backslash A \cap H) \leq 2$, we get $A \cap H=\emptyset$ and $\#\left(\pi_{2}(A)\right)=1$. Set $\{M\}:=\left|\mathcal{I}_{A}(0,1)\right|$. Since $S \nsubseteq M$, Lemma 2.2 gives $h^{1}\left(\mathcal{I}_{B \backslash B \cap H}(1,0)\right)>0$. Thus either there are $b, b^{\prime} \in B \backslash B \cap H$ such that $b \neq b^{\prime}$ and $\pi_{1}(b)=\pi_{1}\left(b^{\prime}\right)$ or $B \cap H=\emptyset$ and $\pi_{1}(B)$ is contained in a line.

Assume the existence of $b, b^{\prime}$. Since $h^{0}\left(\mathcal{O}_{Y}(1,0)\right)=4$, we get the existence if $D \in\left|\mathcal{O}_{Y}(1,0)\right|$ contained $B$ and a point of $A$. Take any $D^{\prime} \in\left|\mathcal{O}_{Y}(0,1)\right|$ such that $D^{\prime} \cap S=\emptyset$. We have $\#\left(\left(D \cup D^{\prime}\right) \cap S\right)=\#(D \cap S) \geq 4$. Since $S$ is a circuit and $\#(D \cap S) \geq 4$, we get $D \cup D^{\prime} \supset S$ and hence $D \supset S$. Since $D$ is a multiprojective space, we get a contradiction. Now assume that $\pi_{1}(B)$ is contained in a line. Since $h^{0}\left(\mathcal{O}_{Y}(1,0)\right)=4$, we get the existence of $D^{\prime \prime} \in\left|\mathcal{O}_{Y}(1,0)\right|$ containing $S$. Since $D^{\prime \prime}$ is a multiprojective space, we get a contradiction.

(d) As in step (c) we exclude all other cases with $k=2$ and $n_{1}+n_{2} \geq 4$. Thus from now on we assume $k \geq 3$.

(e) See Lemma 5.8 for the description of the case $k=3$ and $n_{1}=n_{2}=n_{3}=1$.

(f) Assume $k=3, n_{1}=2$ and $n_{2}=n_{2}=1$. Fix $a, a^{\prime} \in S$ such that $a \neq a^{\prime}$. Take $H \in\left|\mathcal{O}_{Y}(1,0,0)\right|$ containing $\left\{a, a^{\prime}\right\}$. Since $S \nsubseteq H H$, Lemma 2.2 gives $h^{1}\left(\mathcal{I}_{S \backslash S \cap H}(0,1,1)\right)>0$. Thus either there are $b, b^{\prime} \in S \backslash S \cap H$ such that $b \neq b^{\prime}$ and $\eta_{1}(b)=\eta_{1}\left(b^{\prime}\right)$ or $\#(S \backslash S \cap H)=3$ and there is $i \in\{2,3\}$ such that $\#\left(\pi_{i}(S \backslash S \cap H)\right)=1$. In the latter case there is $M \in\left|\mathcal{O}_{Y}\left(\varepsilon_{i}\right)\right|$ containing 
$S \backslash S \cap H$. Since $S \nsubseteq M$, Lemma 2.2 gives $h^{1}\left(\mathcal{I}_{S \backslash S \cap M}\left(\hat{\varepsilon}_{i}\right)\right)>0$. Thus \# $\left.S \backslash S \cap M\right)=2$; that is, $S \backslash S \cap M=\left\{a, a^{\prime}\right\}$ and $\eta_{i}(a)=\eta_{i}\left(a^{\prime}\right)$. In particular we have $\pi_{1}(a)=\pi_{1}\left(a^{\prime}\right)$. Thus there is $H^{\prime} \in\left|\mathcal{O}_{Y}(1,0,0)\right|$ containing $\left\{a, a^{\prime}\right\}$ and at least another point of $H$. Using $H^{\prime}$ instead of $H$ we exclude this case, but not the existence of $b, b^{\prime}$ for $S \backslash H^{\prime} \cap S$, since \# $\left(S \backslash S \cap H^{\prime}\right) \leq 2$.

Now assume the existence of $b, b^{\prime} \in S \backslash S \cap H$ such that $b \neq b^{\prime}$ and $\eta_{1}(b)=\eta_{1}\left(b^{\prime}\right)$. Thus there is $D \in\left|\mathcal{O}_{Y}(0,0,1)\right|$ containing $\left\{b, b^{\prime}\right\}$. Using Lemma 2.2 we get $h^{1}\left(\mathcal{I}_{S \backslash S \cap D}(1,1,0)\right)>0$. Thus one of the following cases occurs:

(1) $S \cap D=\left\{b, b^{\prime}\right\}, \pi_{1}(S \backslash S \cap D)$ is contained in a line and \# $\left(\pi_{2}(S \backslash S \cap D)\right)=1$;

(2) there are $x, y \in S \backslash S \cap D$ such that $x \neq y$ and $\eta_{3}(x)=\eta_{3}(y)$.

First assume $S \cap D=\left\{b, b^{\prime}\right\}, \pi_{1}(S \backslash S \cap D)$ is contained in a line and \# $\left(\pi_{2}(S \backslash S \cap D)\right)=1$. There is $T \in\left|\mathcal{O}_{Y}\left(\varepsilon_{2}\right)\right|$ containing $S \backslash S \cap D$. Lemma 2.2 gives $h^{1}\left(\mathcal{I}_{\left\{b, b^{\prime}\right\}}(1,0,1)\right)>0$. Thus $\eta_{2}(b)=\eta_{2}\left(b^{\prime}\right)$. Since $\eta_{1}(b)=\eta_{1}\left(b^{\prime}\right)$, we get $b=b^{\prime}$, a contradiction.

Assume the existence of $x, y$ such that $x \neq y$ and $\eta_{3}(x)=\eta_{3}(y)$. Write $S=\left\{b, b^{\prime}, x, y, v\right\}$. There is $W \in\left|\mathcal{O}_{Y}(1,0,0)\right|$ containing $b$ and $x$ and hence containing $y$. By Lemma 2.2 we get $h^{1}\left(\mathcal{I}_{S \backslash W}(0,1,1)\right)>0$. Since $\#(S \backslash S \cap W) \leq 2$, we get $S \backslash S \cap W=\left\{b^{\prime}, v\right\}$ and $\eta_{1}\left(b^{\prime}\right)=\eta_{1}(v)$. Using $D^{\prime} \in\left|\mathcal{O}_{Y}(0,1,0)\right|$ containing $\left\{b, b^{\prime}\right\}$ instead of $D$ we get the existence of $x^{\prime}, y^{\prime} \in\{x, y, v\}$ such that $\eta_{2}\left(x^{\prime}\right)=\eta_{2}\left(y^{\prime}\right)$ and $x^{\prime} \neq y^{\prime}$. We have $\left\{x^{\prime}, y^{\prime}\right\} \cap\{x, y\} \neq \emptyset$. With no loss of generality we may assume $x=x^{\prime}$. Either $y^{\prime}=y$ or $y^{\prime}=v$. If $y^{\prime}=y$, we get $x=y$, a contradiction. Thus $\eta_{2}(v)=\eta_{2}(y)$. Hence $\pi_{1}(x)=\pi_{1}(y)=\pi_{1}(v)$. Thus there is $W^{\prime} \in\left|\mathcal{O}_{Y}(1,0,0)\right|$ containing at least 4 points of $S$. Take a general $W_{1} \in\left|\mathcal{O}_{Y}(0,1,1)\right|$. Thus $S \cap W_{1}=\emptyset$. Since $h^{1}\left(\mathcal{I}_{S}(1,1,1)\right)>0$ and $W^{\prime} \cup W_{1}$ contains at least 4 points of $S$, we get $S \subset W^{\prime} \cup W_{1}$. Thus $S \subset W^{\prime}$. Since $W^{\prime}$ is a proper multiprojective space of $Y$, we get a contradiction.

(g) Step (e) excludes all cases with $k \geq 3$ and $n_{i} \geq 2$ for at least one $i$.

(h) Assume $k=4$ and $n_{i}=1$ for all $i$. Fix $o \in S$ and let $H$ be the only element of $\left|\mathcal{O}_{Y}\left(\varepsilon_{4}\right)\right|$ containing $o$. Since $H$ is a multiprojective space, we have $S \nsubseteq H$. Thus Lemma 2.2 gives $h^{1}\left(\mathcal{I}_{S \backslash S \cap H}\left(\hat{\varepsilon}_{4}\right)\right)>0$. Thus one of the following two cases occurs:

(1) $\#\left(\eta_{4}(S)\right)=4$ and $h^{1}\left(Y_{4}, \mathcal{I}_{\eta_{4}(S \backslash S \cap H)}(1,1,1)\right)>0$;

(2) there are $u, v \in S \backslash S \cap H$ such that $u \neq v$ and $\eta_{4}(u)=\eta_{4}(v)$.

(h1) Assume that case (1) occurs. In this case $S \backslash S \cap H=S \backslash\{o\}$. By Proposition 5.2 there is an integer $i \in\{1,2,3\}$ such that $\#\left(\pi_{i}(S \backslash\{o\})=1\right.$. Thus there is $M \in\left|\mathcal{O}_{Y}\left(\varepsilon_{i}\right)\right|$ containing $S \backslash\{o\}$. Take $W \in\left|\mathcal{O}_{Y}\left(\hat{\varepsilon}_{4}\right)\right|$ such that $W \cap S=\emptyset$. Since $H \cup W$ is an element of $\left|\mathcal{O}_{Y}(1,1,1,1)\right|$ containing at least 4 points of $S$ and $h^{1}\left(\mathcal{I}_{S}(1,1,1,1)\right)>0$, we have $S \subset H \cup W$. Since $S \cap W=\emptyset$, we get $S \subset H$, a contradiction.

(h2) By step (h1) there are $u, v \in S \backslash S \cap H$ such that $\eta_{4}(u)=\eta_{4}(v)$. For any $a \in S$ let $H_{a}$ be the only element of $\left|\mathcal{O}_{Y}\left(\varepsilon_{4}\right)\right|$ containing $a$. Thus $H_{o}=H$. By step (h1) applied to $a$ instead of $o$ there are $u_{a}, v_{a} \in S \backslash S \cap H_{a}$ such that $u_{a} \neq v_{a}$ and $\eta_{4}\left(u_{a}\right)=\eta_{4}\left(v_{a}\right)$. Set $E:=\cup_{a \in S}\left\{u_{a}, v_{a}\right\}$.

Claim 1: \# $(E)>2$ and, for all $a, b \in S$, either $\left\{u_{a}, v_{a}\right\}=\left\{u_{b}, v_{b}\right\}$ or $\left\{u_{a}, v_{a}\right\} \cap\left\{u_{b}, v_{b}\right\}=\emptyset$.

Proof: Assume $\#(E) \leq 2$, that is, $\left\{u_{a}, v_{a}\right\}=\left\{u_{b}, v_{b}\right\}$. Since $x \notin\left\{u_{x}, v_{x}\right\}$, taking $b=u_{u_{a}}$ we get a contradiction. Fix $a, b \in S$ such that $a \neq b$ and assume $\#\left(\left\{u_{a}, v_{a}\right\} \cap\left\{u_{b}, v_{b}\right\}\right)=1$, say $\left\{u_{a}, v_{a}\right\} \cap\left\{u_{b}, v_{b}\right\}=\left\{u_{a}\right\}$. Taking $x:=u_{a}, y:=v_{a}$ and $z:=v_{b}$, find $x, y, z \in S$ such that $\#(\{x, y, z\})=3$ and $\nu(\{x, y, z\})$ is contained in a line of of $\nu(Y)$. Thus $S$ is not a circuit, a contradiction.

The first assertion of Claim 1 gives $\#(E)>2$. Then the second assertion of Claim 1 gives $\#(E) \geq 4$. There is $M \in\left|\mathcal{O}_{Y}\left(\varepsilon_{1}\right)\right|$ containing $E$. Since $S \nsubseteq M$, we first get $\#(E)=4$ and then, by Lemma $2.2, h^{1}\left(\mathcal{I}_{S \backslash S \cap M}(0,1,1,1)\right)>0$, contradicting the global span of $\mathcal{O}_{Y}(0,1,1,1)$.

(i) Steps (g) and (h) exclude all cases with $k \geq 4$. 


\section{REFERENCES}

[1] E. Ballico, A. Bernardi, M. Christandl and F. Gesmundo, On the partially symmetric rank of tensor products of $W$-states and other symmetric tensors, Rend. Lincei Mat. Appl. 30 (2019), 93-124,

[2] L. Chiantini, and G. Ottaviani, On generic identifiability of 3-tensors of small rank, SIAM J. Matrix Anal. Applic. 33 (2012), 1018-1037.

[3] L. Chiantini, G. Ottaviani, and N. Vanniuwenhoven, An algorithm for generic and low-rank specific identifiability of complex tensors, SIAM J. Matrix Anal. Applic. 35 (2014), 1265-1287.

[4] L. Chiantini, G. Ottaviani, and N. Vanniuwenhoven, Effective criteria for specific identifiability of tensors and forms, SIAM J. Matrix Anal. Applic. 38 (2017), 656-681.

[5] I. Domanov, and L. De Lathauwer, On the uniqueness of the canonical polyadic decomposition of third-order tensors-part I: Basic results and uniqueness of one factor matrix, SIAM J. Matrix Anal. Appl. 34 (2013), $855-875$.

[6] I. Domanov, and L. De Lathauwer, On the uniqueness of the canonical polyadic decomposition of third-order tensors-part II: Uniqueness of the overall decomposition, SIAM J. Matrix Anal. Appl. 34 (2013), 876-903.

[7] I. Domanov, and L. De Lathauwer, Generic uniqueness conditions for the canonical polyadic decomposition and INDSCAL, SIAM J. Matrix Anal. Applic. 36 (2015), 1567-1587.

[8] T. Graber, J. Harris, J. Starr et A. J. de Jong, Families of rationally connected varieties, J. Amer. Math. Soc. 16 (2003), no. 1, 57-67.

[9] R. Hartshorne, Algebraic Geometry, Springer, Berlin, 1977.

[10] J. W. P. Hirschfeld and J. A. Thas, General Galois Geometries, Oxford Mathematical Monographs, Oxford Science Publications, Clarendon Press, Oxford, 1991.

[11] J. B. Kruskal, Three-way arrays: rank and uniqueness of trilinear decompositions, with application to arithmetic complexity and statistics, Linear Algebra Appl. 18 (1977), no. 2, 95-138.

[12] J. M. Landsberg, Tensors: Geometry and Applications Graduate Studies in Mathematics, Amer. Math. Soc. Providence, 128 (2012).

[13] D. Sacchi, Postulazione di punti su varietà di Segre, Master's thesis, Università di Siena, Advisor L. Chiantini, 2012-2013.

[14] C. Segre, Sulle varietà che rappresentano le coppie di punti di due piani o spazi, Rend. Circ. Mat. Palermo 5 (1891), 192-204; reprinted in Opere, Vol. I, pp. 173-184, Cremonese, Roma, 1957.

Dept. of Mathematics, University of Trento, 38123 Povo (TN), Italy

E-mail address: ballico@science.unitn.it 